\title{
Exploiting Semantic Clustering in the eDonkey P2P Network
}

\author{
S. Handurukande $\dagger$, A.-M. Kermarrec $\ddagger$ F. Le Fessant* \& L. Massoulié \\ $\dagger$ Distributed Programming Laboratory, EPFL, Switzerland \\ $\ddagger$ INRIA, Rennes, France \\ * INRIA-Futurs and Lix, Palaiseau, France \\ * Microsoft Research, Cambridge, UK
}

\section{Abstract}

Peer-to-peer file sharing now represents a significant portion of the Internet traffic and has generated a lot of interest from the research community. Some recent measurements studies of peer-to-peer workloads have demonstrated the presence of semantic proximity between peers. One way to improve performance of peer-to-peer file sharing systems is to exploit this locality of interest in order to connect semantically related peers so as to improve the search both in flooding- and server-based systems. Creating these additional connections raises interesting challenges and in particular (i) how to capture the semantic relationship between peers (ii) how to exploit these relationships and (iii) how to evaluate these improvements. In this paper, we evaluate several strategies to exploit the semantic proximity between peers against a real trace collected in November 2003 in the eDonkey 2000 peer-to-peer network. We present the results of this evaluation which confirm the presence of clustering in such networks and the interest to exploit it.

\section{Introduction and background}

File sharing peer-to-peer systems have significantly grown the past decade to the extent that they are now the highest consumers of Internet bandwidth $[11,14,16]$. As expected, this has generated a lot of interest from the research community to understand these applications, measure their workloads, propose new architectures $[15,20]$ and improve existing ones [7].

Peer-to-peer file sharing systems usually either rely on flat overlays such as Gnutella [3] in which the primary search algorithm is based on flooding, or hierarchical overlays composed of a set of servers indexing peers contents and in charge of redirecting requests. Examples of such hierarchical systems are KaZaA [4] and eDonkey [1]. Besides, number of research works have been done to improve the search mechanism by optimizing replication strategies [13] or switching from flooding to random walks [7]. Recent works $[6,12]$ propose to build Gnutella-like systems in combination with structured peer-to-peer overlays.

More recently, another class of research aims at capturing and exploiting semantic proximity or interest-based proximity to improve the search in peer-to-peer file sharing systems. Semantic proximity between peers is defined as the similarities between their cache contents or download patterns. The idea behind these works is that semantically related peers are more likely to be useful to each other (e.g., than random peers). Therefore, it might be useful to connect semantically related peers in the overlay. These peers, called semantic neighbours in the rest of this paper, are solicited first in the search process because the probability that they are able to satisfy a request might be quite high. In other words, in Gnutella style search schemes the semantic neighbours are queried first; if this first phase fails the normal gossip style phase is performed. In hierarchical schemes like in KaZaA the first phase can be used to bypass the servers (aka super peers) thus alleviating their load.

There are several ways of capturing the semantic relationship between peers. One approach is to explicitly identify distinct semantic groups of documents, using a predefined classification and to build separate overlays for each separate semantic relationship [8]. Precise classification is always a difficult task and the permanent classification may lead to a static configuration which cannot adapt to changes in peers preferences or recovers from a wrong classification. At the other end of the spectrum, in [19], semantic shortcuts are dynamically created to link peers sharing some interests. The semantic relationship between peers is captured implicitly based on the most recent downloads. In [21], alternative ways of creating shortcuts are proposed and evaluated in the context of a synthetic workload.

In a recent paper [9], we presented a clustering analysis of peer-to-peer file sharing traces that we obtained crawling the eDonkey network during a three 
days period. This preliminary study demonstrated that there is a significant overlap between peers contents that one can consider as semantic proximity between peers. In this paper, we evaluate several strategies using implicit or explicit semantic relationships. We evaluate these approaches using the eDonkey 2000 trace we obtained in November 2003. The rest of this paper is organized as follows; in Section 2, we briefly present the experimental setting including the description of the trace; in Section 3, we present the evaluation of three simple strategies (i) implicit semantic; (ii) semantic overlay, and (iii) light explicit semantic, before concluding in Section 4.

\section{Experimental setting}

\subsection{Peer-to-peer workload}

One of the main challenges to validate improvements in peer-to-peer systems is to be able to use realistic input data. In the last few years some measurements studies have been carried out $[17,18,5,10]$ mainly concerned by peer requests, connectivity and availability. Therefore, the associated traces did not include content of the peers (the offered files to the other peers) that we need to carry out our evaluations.

In a recent study [9], we collected and analyzed a peer-to-peer file sharing application trace, focusing on the clustering properties of the peers. To this end, we actively probed a community of the eDonkey 2000 clients, the main competitor of KaZaA, recently referred as ahead of KaZaA in Europe [22]. We obtained a trace of 12,000 clients, sharing 923,000 documents, distributed worldwide with a majority in Europe. We obtained a trace containing for each client, its list of cache contents. In our analysis, we measured files popularity as the number of replicas over the peers' caches. An interesting observation is that the popularity distribution of the files is similar whether it is related to the number of requests [10] or the number of replicas. The study presented in [10] concluded on a fetch once behavior: peers tend to download files only once and therefore do not exhibit temporal locality in their access patterns.

Given this combination of observations, we concluded that a list of cache contents can reasonably be used as a list of requests as explained bellow.

\subsection{Simulation environment}

We wrote a simple discrete-event simulator composed of $n$ nodes to simulate the file exchange between peers and search only using semantic neighbours in a peer- to-peer file sharing system ${ }^{1}$. We assigned nodes randomly to the eDonkey clients of the trace. The simulator maintains the global list of the files shared in the system. Each client is associated with a list of files according to the real trace. The simulation consists in, for each client, requesting sequentially each file of its list. Two situations may occur when a node $n$ requests a file $f$ : (i) either $f$ has already been requested in the system and is replicated in other peers caches. In that situation, $n$ downloads $f$ from one of those peers and updates its semantic neighbours list accordingly (as we will expand in Section 3). If more than one peer has the file, all those peers are taken into account when updating the list. (ii) $n$ is the first peer requesting $f$ and we then consider that $n$ maintains the initial replica of $f$.

\section{Simulating semantic links}

\subsection{Implicit semantic neighbours}

In the first set of experiments, we evaluate two of the strategies described in [21]. To this end, we computed the hit ratio due to the use of semantic neighbours as opposed to random peers. We implemented both the history and LRU strategies. Each peer maintains a list of semantic neighbours, updated during the simulation as follows:

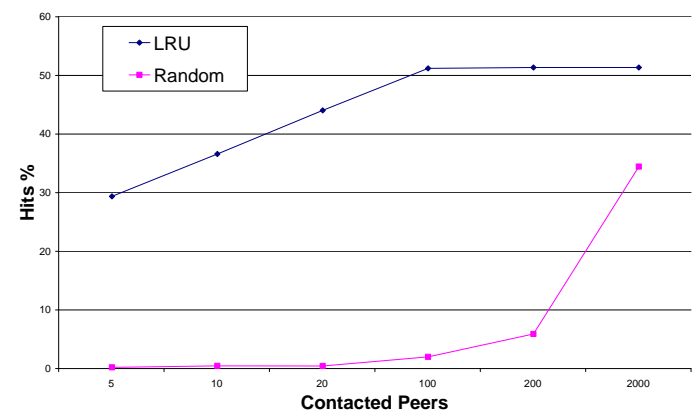

Figure 1: Hit rate with LRU

LRU. The most natural strategy to capture recent history (which proved to work pretty well in data management systems, such as caches for example) is to order the semantic neighbours such that the most recent uploader ${ }^{2}$ peer is placed at the top of the list and to remove the least recent one if needed. This

\footnotetext{
${ }^{1}$ The search algorithm is orthogonal to the way we use the semantic neighbours so we did not implement the search algorithm.

${ }^{2}$ uploader is a peer who offered a desired file to another peer
} 


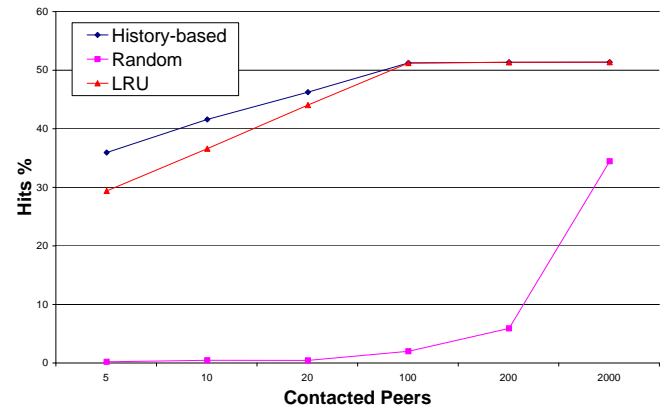

Figure 2: Hit rate, history

method is mostly used in [19] and in [21]. Upon a request, the $x$ ( $x$ is a system parameter) first neighbours of the list are requested first. The standard search mechanism is then used in case of a miss.

History. This strategy has first been introduced in [21]. This approach aims at capitalizing on a larger period of history. Where the LRU strategy selects the $x$ most recent useful peers, History selects the $x$ most useful peers over a time frame. To this end, a peer $i$ maintains an entry for a set of peers depicting how many times the peer $i$ has downloaded a document from other peers with whom peer $i$ previously interacted with. In other words, each peer (with whom peer $i$ previously interacted) is associated with a counter; the peer $i$ increments the counter of peer $j$ whenever $i$ downloads a file from $j$. The implementation of this strategy presents several issues. First of all, the data structure associated with each peer for this purpose grows linearly with the number of uploaders. However, such a data structure might be leveraged by other mechanisms such as incentives [2]. Secondly, deciding on a relevant period is not straightforward: if the period is too long, the strategy will not be able to adapt to change of preferences or interests; on the other hand, if the period is too short, the benefit of using history over LRU is not clear.

Figure 1 displays the hit rate depending on the number of semantic neighbours contacted (as specified by the parameter $x$ ). For the purpose of comparison, the hit rate when choosing the same number of random neighbours is also shown. We observe that the results are very encouraging: even with as few as 5 peers detected as semantically related based on recent downloads, there is a hit rate of close to $30 \%$. Note that the experiment starts with an empty semantic neighbours lists. On the contrary, the hit rate when neighbours are selected randomly is very low and remains low even with a significant number of peers.

Figure 2 shows the hit ratio for the same configuration using History (for the purpose of comparison we also plot the hit ratio of LRU). Results show that history gives a slightly better hit ratio in the context of this trace. However, the fact that in our trace we do not capture any notion of time, we do not detect any dynamic changes in user preferences.

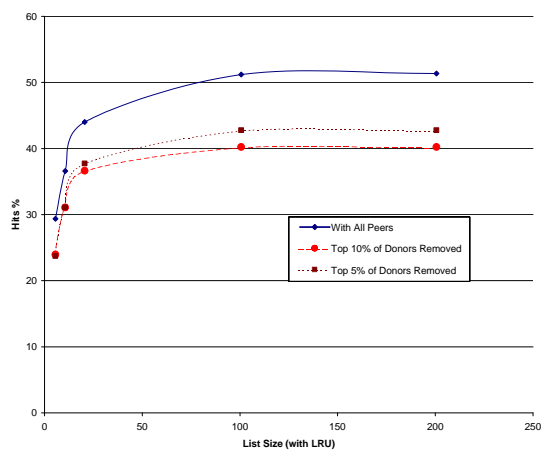

Figure 3: Impact of the removal of the $x \%$ more generous uploaders

The observed improvement in the hit ratio suggests that there is indeed some semantic proximity which is implicitly captured and exploited by the LRU and History strategies. However, the presence of generous uploaders could confuse the semantic relationships. If a few peers provide most of the contents, which has been usually observed in measurements studies, it might be the case that the semantic neighbour lists are mostly populated with these peers. To evaluate the impact of generous peers, we conducted a complementary experiment where we removed from the semantic neighbour lists the $u \%$ most generous uploaders, where we experimented with $u=5 \%, 10 \%, 15 \%$. Figure 3 shows the results. We observed that the hit ratio drops roughly by $6 \%$. This is an indication that the hit ratio is influenced by the presence of very generous uploaders who contribute a large amount of files. In other words, the semantic neighbour lists contain the IDs of very generous peers. But as seen in Figure 3, when the percentage of $u$ is increased from 5 to 15 the reduction of hit ratio keeps unchanged for smaller list sizes (e.g., 5, 10, 20). This indicates that beside the generous peers syndrome, the semantic clustering truly exists. Otherwise the hit ratio would have been decreased linearly as the $u$ is increased. On the other hand, even if the semantic lists contain the IDs of very generous peers, contact- 
ing peers in the list pays off well since the approach allows to bypass the normal search mechanism (either gossip-based or server-based) which is generally very expensive due to flooding or due to heavily loaded servers.

\subsection{Semantic overlay}

In the second set of experiments, we investigate the transitivity of the semantic relationship. Is it true that the semantic neighbours of my semantic neighbours are my semantic neighbours?. To this end, we extended the search to two hops away, using the semantic neighbours. More precisely, first a peer searches using its semantic neighbours; failing that, the peer uses the semantic neighbours of its semantic neighbours by forwarding the search query on an overlay network made up of semantic neighbours. Results are depicted on Figure 4. For comparison, we plot the results obtained according to the list size (as specified by $x$ ) of semantic neighbours for both direct semantic neighbours and 2nd level semantic neighbours which are 2 hops away. In other words, the amount of memory used to keep the information about semantic neighbours at each peer is fixed for 2 schemes; but for a given size of the list (or $x$ ) the number of nodes contacted while searching is much higher (in the worst case scenario) when the 2nd level of semantic neighbours are also used. For example, when the list size is 5 , in the worst case scenario, 25 peers are contacted in the 2 nd level semantic search.

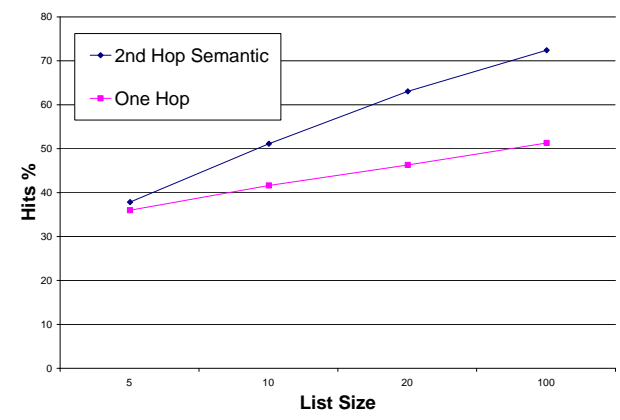

Figure 4: Semantic overlay

Results do not exhibit better results for 2nd level semantic search in particular, if we consider that the important factor is the number of peers contacted. However, the fact that 2nd-level semantic search provides similar results as when the same number of semantic neighbours peers are contacted during the first hop, shows that semantic links tend to automat- ically cluster semantically-related peers.

\subsection{Explicit semantic}

As we observed in previous experiments, simple heuristic enables to capture implicit semantic relationships between peers (without any additional information on the content exchanged or the peer profiles) and to cluster peers together. On the other hand, the information about the type of file being searched and peer profiles (such as the content they store) can be used to make the semantic search more efficient.

We evaluated one such light explicit semantic scheme. To that end, instead of maintaining a unique list of semantic neighbours, each peer maintains several lists (according to the LRU or History policy), for example one per type of the file (audio, video, software etc). In other terms, each type is associated with a separate list of semantic neighbours and a peer contacts only the neighbours who are in the relevant list for a given search. eDonkey, as well as most of its other competitors, associate with each file some meta information about the file such as the name, size, file extension and the kind of file (audio, video etc). These meta information helps when devising these explicit semantic schemes. In this last set of experiments, we investigate the use of already available information in most peer-to-peer file sharing systems and Figure 5 depicts the hit ratio obtained for audio files using both implicit and explicit semantics. These results show that the hit ratio is impacted when maintaining a peer profile per type of file.

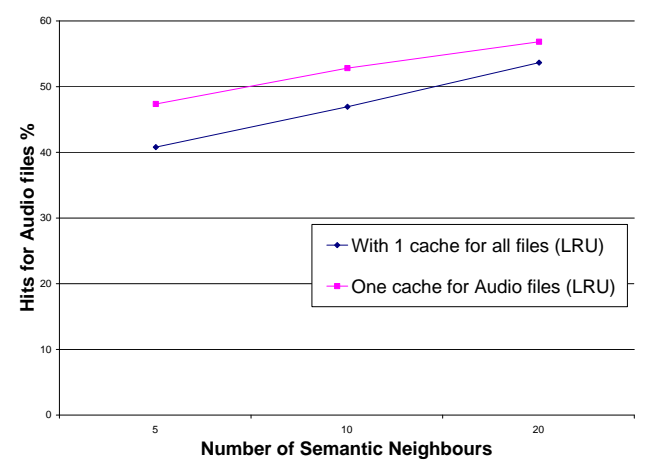

Figure 5: Explicit versus implicit

Another important point to note is that in the implicit scheme, while searching files that are in different categories of interests, a peer might lose (e.g., due to pruning of lists) some semantic neighbours just because those neighbours have no relevance to 
the current search or interests. This can be avoided by having separate lists for each interest group.

One could argue that the number of links maintained is much higher. This is true but that the important factor in terms of load is the number of neighbours which are contacted on a request rather than the number of pointers maintained on each node ${ }^{3}$. In addition, given the fact that a peer can have number of interest categories and different peers will act as uploaders for different categories, it is natural to think that a peer may exhibit semantic proximity with different peers depending on the type of information.

\section{Conclusion}

In this paper, we evaluated some simple strategies that can easily capture and exploit the semantic relationships observed between peers in peer-to-peer file sharing systems. The main issue in semantic proximity is how to capture the semantic relationship between two peers without explicitly involving peers themselves or structuring or grouping peers in the overlays into a static configuration which 1) does not evolve well as the interests of peers change and 2) generally needs significant amount of manual intervention by the users when structuring the peers.

We evaluated two different policies (LRU and History based) of maintaining semantic neighbours according to implicit scheme and present a simple explicit scheme. As was shown both schemes produce good results in terms of hit ratio, demonstrating that exploiting the observed clustering between peers may lead to improvements in the search process. One important property of such improvements is that the approach may be used in unstructured (floodingbased), semi-structured (super-peers-based) or structured (DHT-based) peer-to-peer file sharing systems. We are currently investigating the implementation in these various contexts. Future work is also needed to evaluate these approaches in dynamic traces.

\section{References}

[1] edonkey. http://www.edonkey2000.com/index.html.

[2] Emule. http://www.emule-project.net/.

[3] Gnutella. http://www.gnutella.com.

[4] KazAa. www.kazaa.com.

[5] R. Bhagwan, S. Savage, and G. Voelker. Understanding availability. In IPTPS'03, Feb. 2003.

\footnotetext{
${ }^{3}$ maintaining semantic peers does not involve a heavy heartbeat-based scheme.
}

[6] M. Castro, M. Costa, and A. Rowstron. Should we build gnutella on a structured overlay? In HotNets 2003, Boston, MA, USA, Nov 2003.

[7] Y. Chawathe, S. Ratnasamy, L. Breslau, N. Lanham, and S. Shenker. Making gnutella-like p2p systems scalable. In SIGCOMM'03, 2003.

[8] A. Crespo and H. Garcia-Molina. Semantic overlay networks for $\mathrm{p} 2 \mathrm{p}$ systems. Technical report, Stanford University, 2003.

[9] F. L. Fessant, S. Handurukande, A.-M. Kermarrec, and L. Massoulié. Clustering in peer-to-peer file sharing workloads. In The 3rd International Workshop on Peer-to-Peer Systems (IPTPS'04), 2004.

[10] K. P. Gummadi, R. J. Dunn, S. Saroiu, S. D. Gribble, H. M. Levy, and J. Zahorjan. Measurement, modeling, and analysis of a peer-to-peer file-sharing workload. In SOSP'03.

[11] http://bitconjurer.org/BitTorrent/. Bittorrent.

[12] B. T. Loo, R. Huebsch, I. Stoica, and J. Hellerstein. The case for a hybrid $\mathrm{p} 2 \mathrm{p}$ search infrastructure. In The 3rd International Workshop on Peerto-Peer Systems (IPTPS'04), 2004.

[13] Q. Lv, P. Cao, E. Cohen, K. Li, and S. Shenker. Search and replication in unstructured peer-to-peer networks. In Proceedings of the 16th international conference on Supercomputing, 2002.

[14] D. Plonka. Napster traffic measurement. Technical report, University of Wisconsin-Madison, 2000.

[15] A. Rowstron and P. Druschel. Pastry: Scalable, distributed object location and routing for large-scale peer-to-peer systems. In Middleware, 2001.

[16] S. Saroiu, K. P. Gummadi, R. Dunn, S. D. Gribble, and H. M. Levy. An analysis of Internet content delivery systems. In OSDI'02, Dec. 2002.

[17] S. Saroiu and S. G. P. Krishna Gummadi. A measurement study of peer-to-peer file sharing systems. In $M M C N^{\prime}$ '02, Jan. 2002.

[18] S. Sen and J. Wong. Analyzing peer-to-peer traffic across large networks. In SIGCOMM'02 Workshop on Internet measurment, 2002.

[19] K. Sripanidkulchai, B. Maggs, and H. Zhang. Efficient content location using interest-based locality in peer-to-peer systems. In INFOCOM'03.

[20] I. Stoica, R. Morris, D. Karger, F. Kaashoek, and H. Balakrishnan. Chord: A scalable peer-to-peer lookup service for internet applications. In $S I G$ COMM 2001, San Diego, USA, Aug. 2001.

[21] S. Voulgaris, A.-M. Kermarrec, L. Massoulié, and M. van Steen. Exploiting semantic proximity in peer-to-peer content searching. In 10th International Workshop on Future Trends in Distributed Computing Systems (FTDCS 2004), China, May 2004.

[22] G. Wearden. eDonkey pulls ahead in European P2P race. http://news.com.com/2100-1025_35091230.html, 2003. 